\title{
Very-high-cycle fatigue behavior of a structural steel with and without induced surface defects
}

\author{
Qingqing Jiang, Chengqi Sun, Xiaolong Liu, Youshi Hong* \\ LNM, Institute of Mechanics, Chinese Academy of Sciences, Beijing 100190, China
}

\section{A R T I C L E I N F O}

\section{Article history:}

Received 14 February 2016

Received in revised form 25 May 2016

Accepted 26 May 2016

Available online 28 May 2016

\section{Keywords:}

Very-high-cycle fatigue

Surface defect

Fine-granular-area

Crack initiation

Structural steel

\begin{abstract}
A B S T R A C T
Fatigue tests via rotary bending were performed on the specimens with and without induced surface defects for a structural steel of medium carbon content to investigate the effect of surface defects on fatigue behavior in high-cycle fatigue and very-high-cycle fatigue (VHCF) regimes. The S-N data showed that induced surface defects substantially degraded the related fatigue strength. For the specimens without surface defects failed in VHCF regime, crack initiated from the interior of specimens with inclusions or matrix inhomogeneities as crack origin and the initiation regions were of different extents of rough surface. The observations on the profile samples cut from crack initiation regions revealed that the region was a layer of nanograins for the case of inclusion as crack origin, and was without grain refinement for the case of grain boundary as crack origin. For the specimens with induced surface defects, crack initiated from surface defects and the initiation region was without grain refinement. The characteristics of crack initiation were carefully examined and the effect of surface defects on fatigue strength degradation was analyzed by available models.
\end{abstract}

(c) 2016 Elsevier Ltd. All rights reserved.

\section{Introduction}

Since the work of Naito et al. [1], very-high-cycle fatigue (VHCF) behavior has received increasing attentions in fatigue research community [2-8]. For high-strength steels, cracks are prone to initiate at specimen subsurface or interior [9-13] in VHCF regime, and a distinct crack initiation feature of "fish-eye" morphology embracing a region of "fine-granular-area (FGA)" usually presents at fracture surface. Another typical feature of VHCF behavior for highstrength steels is that the S-N curve is a stepwise or duplex shape [14-21] with regard to surface-induced and interior-induced crack initiation, respectively. Bathias and Paris [22] investigated several high-strength steels via ultrasonic fatigue test technique and revealed that subsurface crack initiated from either nonmetallic inclusions or other microstructural inhomogeneities. They gave an explanation: the probability of a sufficient stress concentration inhomogeneity is much higher in the interior of material than that at the surface. Chai [13] reported that the formation of subsurface non-defect fatigue crack was caused by a material damage process of soft phase due to cyclic plastic deformation. Yang et al. [23] measured the FGA of two spring steels and proposed a criterion to estimate the size of FGA. Hong et al. [24] performed tests of a

\footnotetext{
* Corresponding author.

E-mail address: hongys@imech.ac.cn (Y. Hong).
}

high carbon chromium steel and calculated fatigue life from FGA to fish-eye and from fish-eye to the critical crack size. Their result demonstrated that the formation of FGA is responsible for a majority part of total fatigue life and the fraction of fatigue life contributed by FGA is beyond $95 \%$ in VHCF regime. Further, a $D^{*}$ parameter has been proposed to discuss the competition of crack initiation from the surface and from the interior of specimen $[20,21]$, which gave an explanation for the preference of fatigue crack initiation either from the surface or from the interior of specimen.

For the formation of FGA, Murakami et al. [25,26] proposed a model of "hydrogen assisted crack growth". It was considered that hydrogen trapped by an inclusion caused the crack growth at a very slow rate, and FGA was a kind of rough fracture surface formed at the crack initiation stage. When the size of FGA reached a critical value, the crack growth proceeded without the assistance of hydrogen. Shiozawa et al. [10] considered that the spherical carbides in steels interspersed around the inclusion would debond first from the matrix and form the microcracks during the fatigue process, and then the microcracks connected to form FGA. The crack propagation was independent of the spherical carbide decohesion after FGA reached a critical size. Sakai $[27,28]$ stated that a fine granular layer was first generated in the vicinity of an inclusion during a large number of cyclic loadings. The microcracks formed by the debonding between the fine granular layer and 
the matrix. Thus, the initiated microcracks connected to each other and ran through the fine granular layer region. A similar explanation of "local grain refinement at the crack tip" [29] was subsequently proposed, which together with the previous one by Sakai $[27,28]$ considered that the fine grains were produced before the related crack formation.

It is noted that Shanyavskiy [30] also proposed a model to explain the formation of FGA, in which it described "Vortical plastic flows ... are creative of a thin nanostructure layer ...", and "The nanoscopic grains experience rotation in each compression/tension half-cycle; thereby the grain-boundary strength (cohesion) diminishes to allow grains partition and to form the free surface of the particles in the FGA zone". Although the physical detail of this model needs to be verified, it presumed nanoscopic particle preexistence in the vicinity of the inclusions and the nanograin generation also before the related crack formation. Recently, Hong et al. [31] revealed that the FGA region is a nanograin layer of several hundred nanometer thick on both sides of crack surface, and proposed a new mechanism of FGA formation that is due to the numerous cyclic pressing (NCP) between the originated crack surfaces associated with crack closure. Note that the experimental cases investigated in Ref. [31] were on high-strength steels with high carbon content. For the cases of structural steels with medium carbon content and the crack initiation from the interior of material with fish-eye morphology, is the microscopic feature of crack initiation region the same with the reported cases of high carbon steels? And is the previous proposed NCP model able to explain the formation process of VHCF crack initiation region for structural steels? These are still unclear questions remained for further investigation.

It is certain that surface defects of specimen have remarkable influence on VHCF behavior. In reality, engineering components always contain more or less surface defects that are unavoidably induced in machining and/or in service. The surface defects with relatively large size are likely to be the fatigue crack origin. The research by Li et al. [32] demonstrated that surface grinding defect induced crack initiation was the predominant failure mode of the steel in VHCF regime. A series of experimental research by Murakami [33] and Lorenzino et al. [34] investigated the effect of surface defects on the fatigue strength at $10^{7}$ cycles with rotary bending fatigue and proposed a formula for predicting the fatigue strength based on a large number of experiments:

$\sigma_{\mathrm{W}}^{6} \sqrt{\text { area }}=C$,

where $\sigma_{\mathrm{W}}$ is fatigue strength, $\sqrt{\text { area }}$ is the square root of defect projection area, and $C$ is a material parameter. Murakami [33] also indicated that surface defect size is the primary parameter, and fatigue strength is nearly independent of defect morphology and tilt angle to the loading axis. Shyam et al. [35] studied the effect of submillimeter size holes on the fatigue limit of a high-strength tool steel via small crack growth tests, and proposed an elastic plastic fracture mechanics method for predicting the fatigue limit of specimens with submillimeter holes. Endo and Ishimoto [36] performed fatigue tests on specimens of two steels and proposed a criterion for predicting the fatigue strength of defect-containing specimens subjected to combined axial and torsional loadings. In practice, the role of surface defects in the impact on fatigue behavior may vary. The research of Fernández-Pariente et al. [37] reported that surface treatment like nitriding and shot peening led to different sensibilities of fatigue resistance to the same surface defect. Morel et al. [38] investigated the competition between microstructure and defect by using FE simulations, and summarized that local stress field is highly scattered and differs significantly from the macroscopic response of the polycrystalline aggregate. The research of Leopold et al. [39] on Ti-6Al-4V alloy obtained a similar result. They stated that fatigue life, from $10^{5}$ to $10^{7}$ cycles, is controlled by crack initiation even for the initiation from defects. Although a number of results have been reported regarding the effect of surface defects, the behavior of crack initiation induced by surface defects (especially in VHCF regime) is still an essential issue requiring further investigation.

In this paper, rotary bending fatigue tests were performed on the specimens with and without induced surface defects of a structural steel with medium carbon content, and the effect of induced surface defects on the fatigue strength was investigated. The fracture surfaces of the specimens with and without induced surface defects were observed with scanning electron microscopy (SEM). The micro-morphologies of crack initiation regions were carefully examined by transmission electron microscopy (TEM) equipped with selected area electron diffraction (SAD) detection unit on the samples prepared by focused ion beam (FIB) cutting. The detail observations revealed that for the specimens without induced surface defects, the FGA region with an inclusion as crack origin is a thin layer of nano-sized grains. For the specimens with induced surface defects, cracks solely initiated from the surface defects even in high-cycle fatigue and VHCF regimes, and the micro morphology of crack initiation region is different from the cases without surface defects. In addition, the effect of surface induced defect on the fatigue strength was evaluated with available models and the result was compared with experimental data in literature.

\section{Test material and experimental methods}

The material used in this paper is a structural steel with medium carbon content, and the chemical compositions (wt.\%) are: $0.42 \mathrm{C}, 0.31 \mathrm{Si}, 0.82 \mathrm{Mn}, 0.0072 \mathrm{P}, 0.0084 \mathrm{~S}, 0.02 \mathrm{Al}, 0.0037 \mathrm{~N}$, $0.0006 \mathrm{O}$ and Fe balance. Two groups of specimens were machined for this investigation. One group of specimens (group A) were heated with high frequency induction heater for $3 \mathrm{~s}$ then quenched in water (BW water base quenching liquid) and tempered for $2 \mathrm{~h}$ at $220^{\circ} \mathrm{C}$. The other group of specimens (group B) were heated at $860^{\circ} \mathrm{C}$ for $10 \mathrm{~min}$ in salt-bath furnace then quenched in nitrate solution at $170^{\circ} \mathrm{C}$ and tempered for $2 \mathrm{~h}$ at $230^{\circ} \mathrm{C}$. Such heattreatment procedures were conducted to ensure the same resultant microstructure and mechanical properties for the two groups. As a result, the microstructure was tempered martensite for the two groups, and the average micro-hardness was $550 \mathrm{Hv}$ (kgf/ $\mathrm{mm}^{2}$ ) for both group A and group B specimens measured by micro-hardness tester at a load of $3 \mathrm{~N}$ with the loading time of $15 \mathrm{~s}$. By considering that the two groups are with the similar microstructure and micro-hardness, just one specimen group (group B before the introduction of surface defects) was chosen for tensile test and the result showed that the average yield strength was $1552 \mathrm{MPa}$ and the tensile strength was $1883 \mathrm{MPa}$ from three cylindrical specimens with the diameter of $5 \mathrm{~mm}$ in gage section.

The shape and dimensions of group A and group B specimens are shown in Fig. 1. Before fatigue testing, the round notch surface was ground and polished for both group A and group B specimens. For each specimen in group B, two surface defects (holes) were introduced in the smallest section symmetrically $\left(180^{\circ}\right.$ in between) with a micro milling machine and such specimens were tempered at $220^{\circ} \mathrm{C}$ for $2 \mathrm{~h}$ to eliminate possible residual stress resulted from the drilling process. The fatigue tests were conducted on a rotary bending machine at a frequency of $52.5 \mathrm{~Hz}$ at room temperature in air with the stress ratio of $R=-1$.

The fracture surface morphologies of all broken specimens were observed by SEM, and the chemical compositions of crack initiation spots (inclusions or other inhomogeneities) were examined by energy dispersive spectroscopy (EDS). Further, profile samples, 


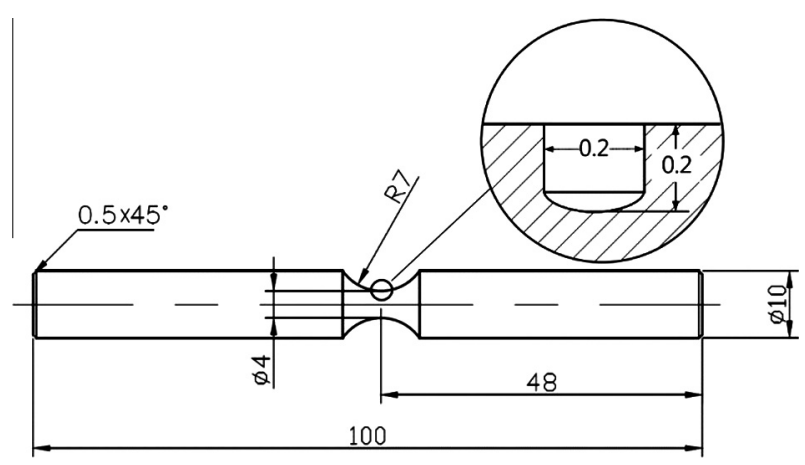

Fig. 1. Shape and dimensions (in $\mathrm{mm}$ ) of group A and B specimens, group A specimen without induced surface hole, and group B specimen with induced surface hole of $200 \mu \mathrm{m}$ in diameter and $200 \mu \mathrm{m}$ in depth; right end of the specimen clamped to rotary bending machine and left end loaded with applied weight.

each with the size of $10 \mu \mathrm{m}$ (width) $\times 5 \mu \mathrm{m}$ (depth) $\times 80 \mathrm{~nm}$ (thickness), were precisely sliced from crack initiation regions with FIB cutting, and the characteristics of crack initiation regions were carefully examined via TEM with the equipped detection unit of $\mathrm{SAD}$. The size of SAD was $200 \mathrm{~nm}$ in diameter for all cases except for specimen A4 for which the size of SAD was $250 \mathrm{~nm}$ in diameter.

\section{Experimental results}

\section{1. $S-N$ data}

Fig. 2 shows the results of S-N data for the two groups of specimens. It is seen that, for group A specimens, the fatigue strength decreases with the increase of fatigue life and the crack tends to initiate from the interior of specimen when the fatigue life beyond $5 \times 10^{6}$. For group B specimens, the fatigue strength is substantially lower than that of group $\mathrm{A}$, and there is a plateau region between $10^{5}$ and $10^{8}$ cycles. Fatigue cracks for all broken specimens in group B initiated from the induced surface defects.

\subsection{Fracture surface morphology}

For group A, crack initiation for most specimens was from the surface of specimen in low-cycle fatigue and high-cycle fatigue regimes, and the typical morphology was illustrated in Fig. 3. In VHCF regime, crack initiation for most specimens was from the

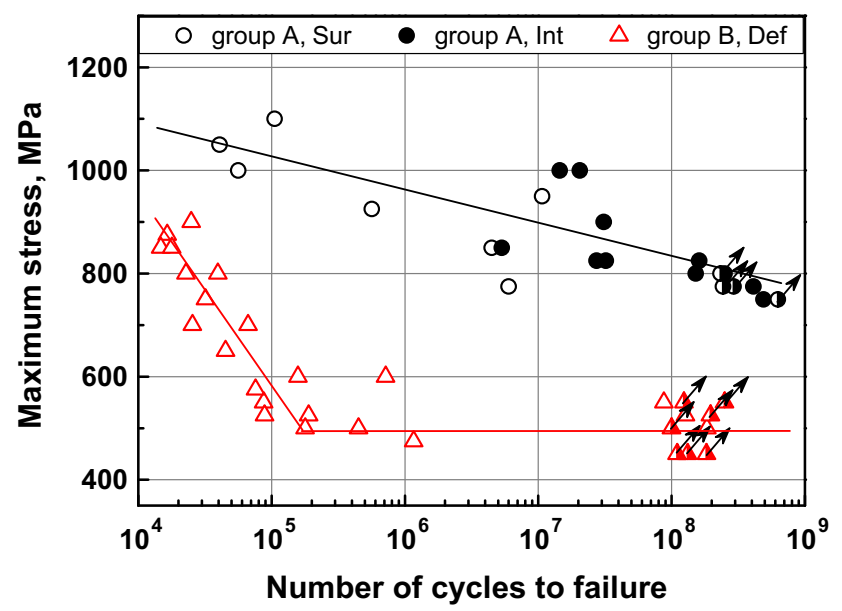

Fig. 2. S-N data for two groups of specimens. Sur: crack initiation from surface, Int: crack initiation from interior, Def: crack initiation from defect, point with arrow: no failure specimen. inclusion in the subsurface of specimen and a typical morphology of fracture surface was shown in Fig. 4. A couple of specimens failed due to crack initiation from grain boundary in the subsurface of specimen in VHCF regime and the typical fractography was shown in Fig. 5. It is evident that for group A specimens, either inclusion initiation mode or grain boundary initiation mode prevailed to result in the characteristic region of fish-eye and FGA morphology in VHCF regime.

Different from the cases of group A described above, the specimens of group B all failed from the induced surface defects from the whole range of low-cycle fatigue to VHCF regimes. Fig. 6 is the fractography of a specimen failed in VHCF regime. It is seen that the crack initiation is from the surface of hole root.

\subsection{Observations of crack initiation profiles}

In order to further investigate the crack initiation characteristics, profile samples were cut from the crack initiation regions of five specimens (four from group A: A1, A2, A3, A4 and one from group B: B1 $)$ via FIB. Specimen A1 $\left(\sigma_{\max }=1000 \mathrm{MPa}, N_{\mathrm{f}}=2.1 \times 10^{7}\right)$ failed from non-metallic inclusion in VHCF regime with clear FGA morphology in crack initiation region. Specimen $A 2\left(\sigma_{\max }=825 \mathrm{MPa}\right.$, $N_{\mathrm{f}}=1.6 \times 10^{8}$ ) failed also from non-metallic inclusion in VHCF regime but with more developed FGA morphology in crack initiation region. Specimen A3 $\left(\sigma_{\max }=850 \mathrm{MPa}, N_{\mathrm{f}}=5.3 \times 10^{6}\right)$ failed from grain boundary in high-cycle fatigue regime with FGA-like morphology in crack initiation region. Specimen $A 4\left(\sigma_{\max }=900\right.$ MPa, $N_{\mathrm{f}}=3.1 \times 10^{7}$ ) failed also from grain boundary with FGA-like morphology in crack initiation region. For specimen B1 $\left(\sigma_{\max }=500 \mathrm{MPa}, N_{\mathrm{f}}=1.8 \times 10^{8}\right)$, the crack initiation was from surface defect without FGA morphology in crack initiation region. The fracture surface morphologies together with the position for FIB sample cutting of five specimens are shown in Fig. 7.

In addition, EDS examinations indicated that the chemical compositions of the inclusion in specimens A1 (Fig. 7a) and A2 (Fig. 7b) primarily consist of $\mathrm{Al}_{2} \mathrm{O}_{3}$. EDS results of crack initiation origin for specimens A3 (Fig. 7c) and A4 (Fig. 7d) are primarily Fe element confirming that the crack initiation is due to the inhomogeneities of the matrix.

Fig. 8 shows the TEM observation result of A1. From Fig. 8a, it is observed that the microstructure in the whole TEM sample was almost common tempered product except for the thin layer underneath the facture surface. The SAD patterns of Fig. 8b-d show a kind of slightly elongated spots for the locations underneath the FGA surface, which is a reflection of a couple of grains within the diffraction area with texture-like feature (by the diffraction principle [40]), whereas in the location away from the FGA surface, the SAD image exhibits a clear pattern of isolated spots (Fig. 8e), implying just one grain (by the diffraction principle [40]) in the diffraction area.

Fig. 9 shows the TEM image and SAD patterns of FIB sample cut from specimen A2. As seen in Fig. 9a, the microstructure beneath the FGA surface is different from that from the inside; the former is a layer of fine grains and the thickness is about several hundred nanometers. The SAD patterns from this layer (Fig. 9b and c) are discontinuous diffraction circles, suggesting that several grains exist within the diffraction area (by the diffraction principle [40]), while the SAD pattern away from the surface (Fig. 9d) shows the feature of single grain diffraction.

Fig. 10 is an example (specimen A3) of TEM image with SAD examinations for crack initiation from grain boundary. It is observed that the microstructure (Fig. 10a) was the tempered product from just underneath the surface to the inside of the specimen. The SAD patterns for the locations underneath FGA-like surface (Fig. 10b-d) or away from the fracture surface (Fig. 10e) are all isolated spots, indicating that the microstructure under- 

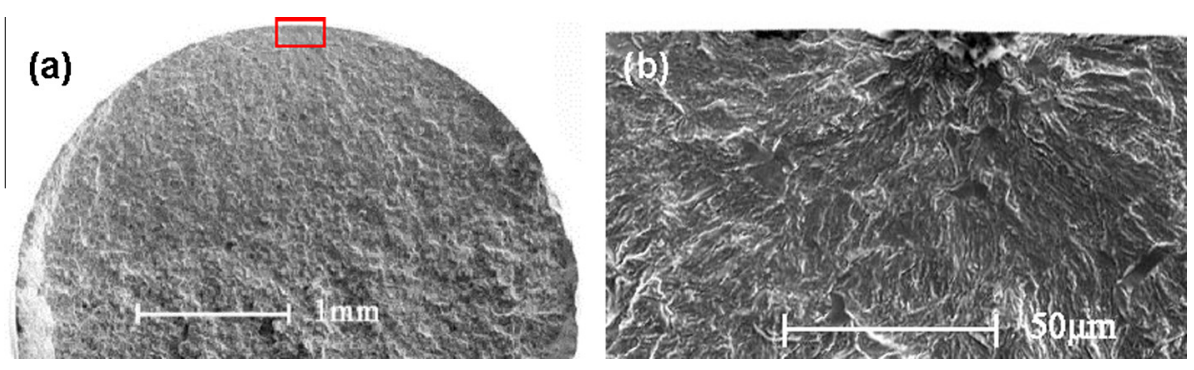

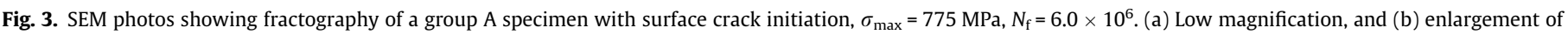
the marked rectangle in (a).
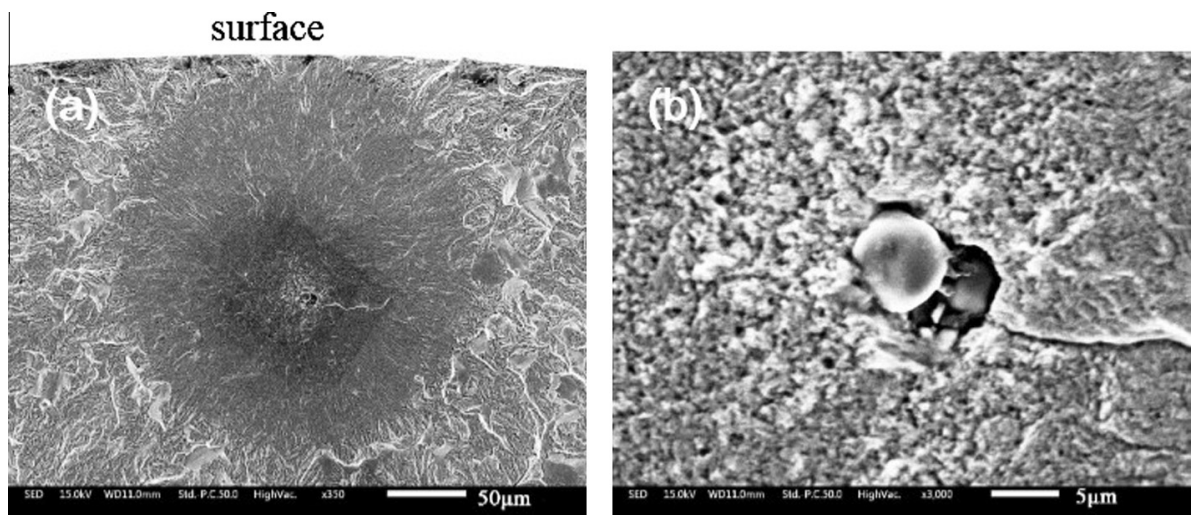

Fig. 4. SEM photos of a group A specimen failed from an inclusion in specimen subsurface, $\sigma_{\max }=825 \mathrm{MPa}, N_{\mathrm{f}}=1.6 \times 10^{8}$. (a) Low magnification showing whole fish-eye, and (b) high magnification for crack origin.
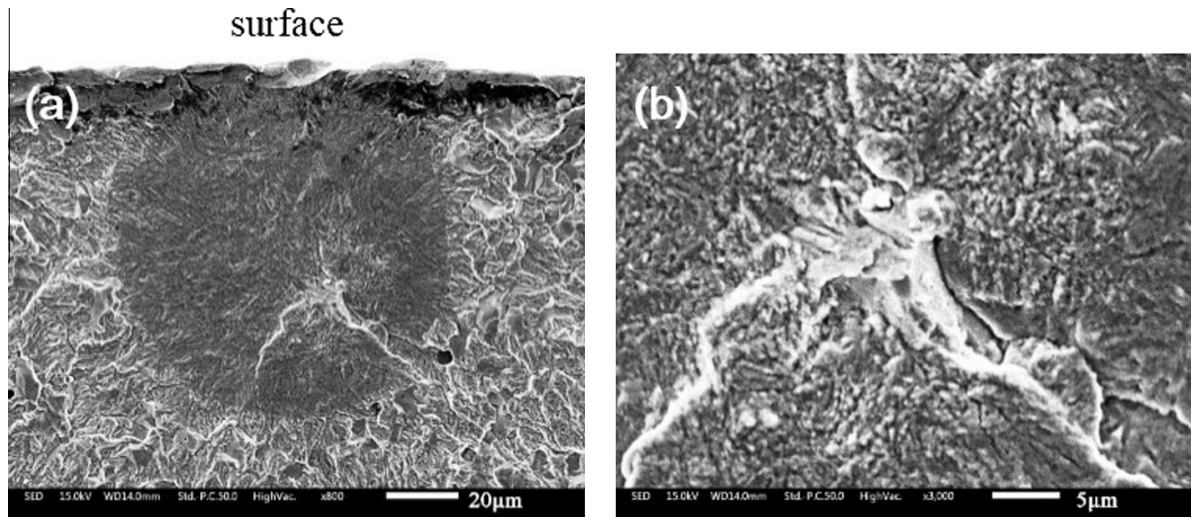

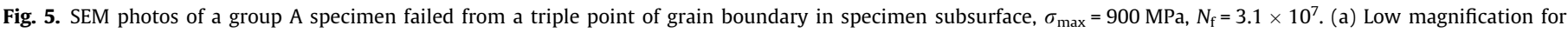
fracture surface with whole fish-eye, and (b) high magnification for crack origin.

neath the fracture surface or away from the fracture surface is the coarse grains of ordinary tempered martensite.

Fig. 11 presents the TEM image with SAD examinations of specimen A4 (crack initiation due to grain boundary), which shows the microstructure image almost consistent at the locations underneath FGA-like fracture surface and inside the specimen. Five SAD examinations (Fig. 11b-f) were performed for the locations from just underneath the fracture surface to away from the surface. For just underneath the surface, Fig. 11c shows one set of isolated spots and Fig. 11e shows two sets of isolated spots. Away from the fracture surface, the SAD examinations of Fig. $11 \mathrm{~b}$, $\mathrm{d}$ and $\mathrm{f}$ are also the patterns of isolated spots. The SAD results of specimen A4 together with those of specimen A3 suggest that for the case of crack initiation from grain boundary in VHCF regime, no evidence of grain refinement exists in the crack initiation region.
Fig. 12 shows the examinations on the sample cut from specimen B1. Fig. 12b and c are two results of SAD examinations just underneath the fracture surface, with Fig. 12b showing two sets of isolated spots and Fig. 12c showing one set of isolated spots. Fig. 12d is an example of SAD result away from the fracture surface, which shows one set of isolated spots. These results indicate that for the case of crack initiation from induced surface defects, no evidence of grain refinement presents in the crack initiation region.

\section{Discussion}

\subsection{Effect of induced surface defect on fatigue strength}

As illustrated in Fig. 2, the presence of induced surface defects leads to a substantial decrease in fatigue strength. For example, 

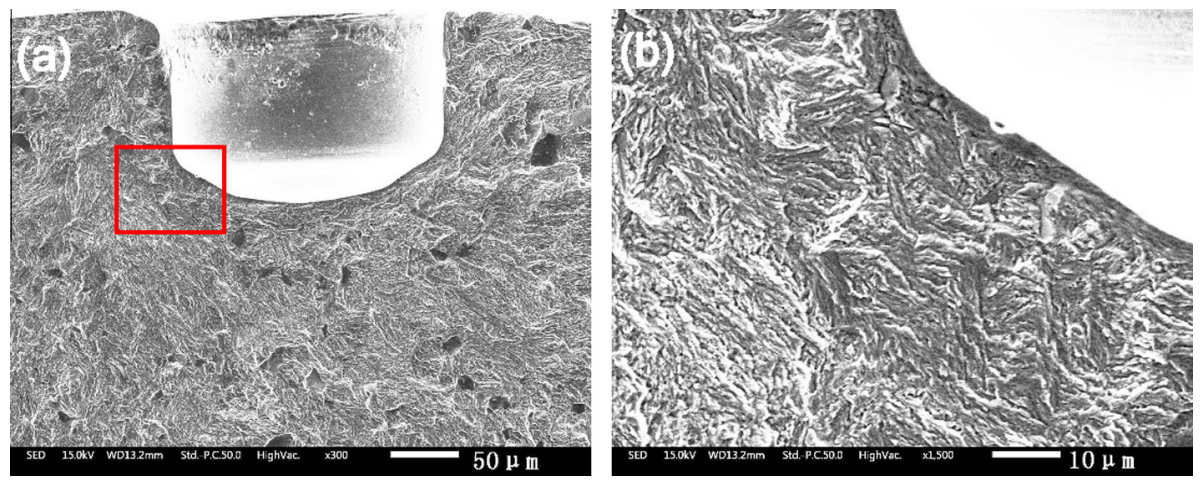

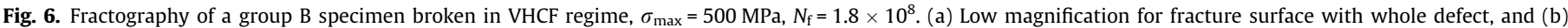
enlargement of the marked rectangle in (a).
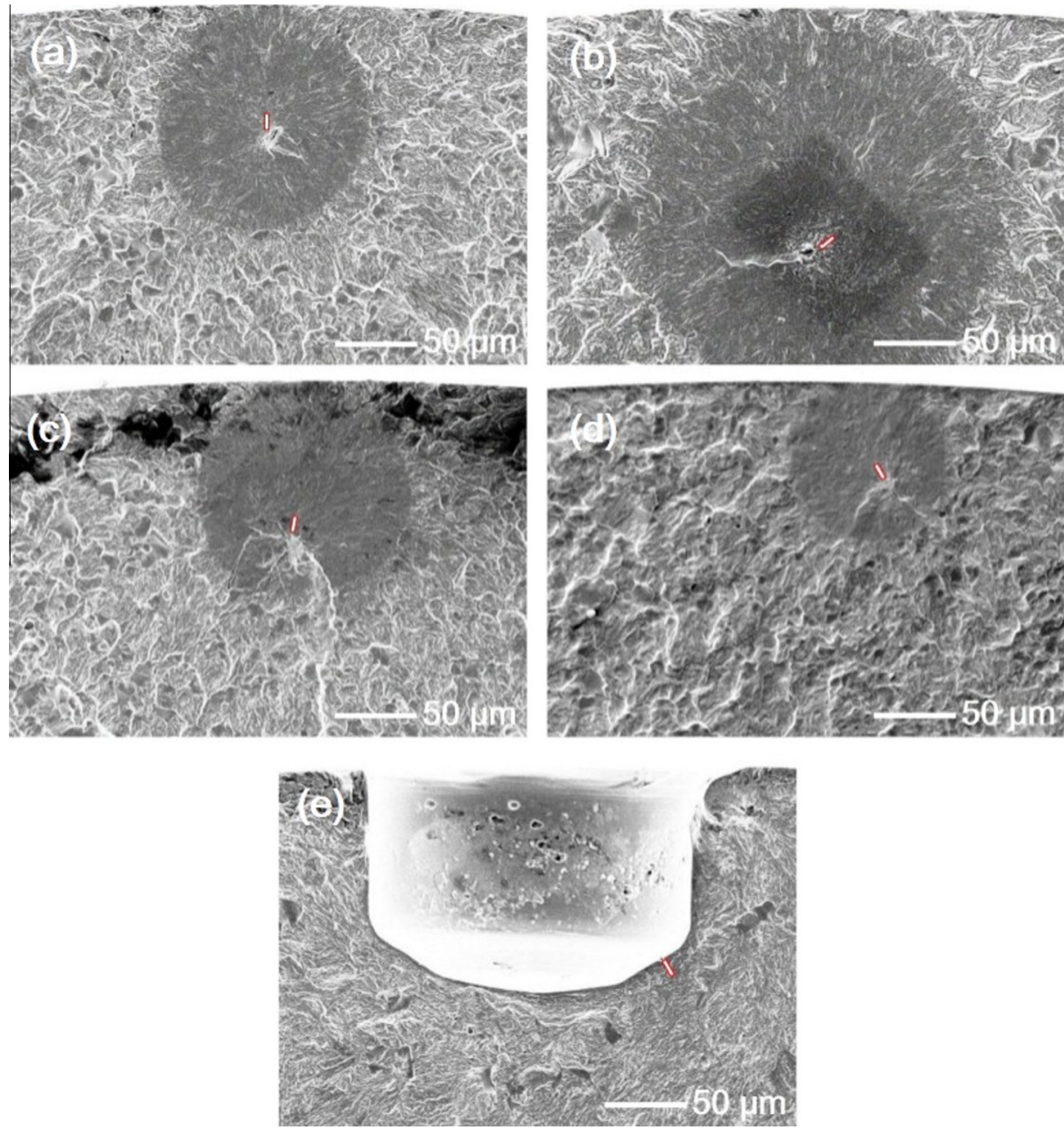

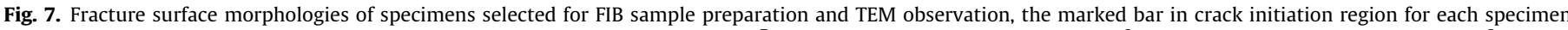

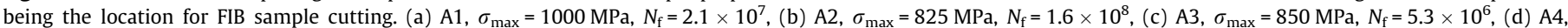
$\sigma_{\max }=900 \mathrm{MPa}, N_{\mathrm{f}}=3.1 \times 10^{7}$, and (e) B1, $\sigma_{\max }=500 \mathrm{MPa}, N_{\mathrm{f}}=1.8 \times 10^{8}$.

at the fatigue life of $10^{7}$ cycles, the fatigue strength decreases by about $41 \%$ for the case with induced surface defects, and it decreases by about $37 \%$ at the fatigue life near $10^{8}$ cycles. The role of induced surface defects in the degradation of fatigue strength can be explained in terms of the related stress intensity factor, i.e. the driving force for crack initiation. With regard to crack initiation, the value of stress intensity factor range at the periphery of inclusion $\left(\Delta K_{\text {Inc }}\right)$ was calculated by the following equation [41]:
$\Delta K_{\mathrm{Inc}}=\alpha \sigma\left(\pi \sqrt{\text { area }}^{1 / 2}\right.$,

where area represents the inclusion projection area, and $\sigma$ is the maximum applied stress (for the case of $R=-1$ ). For the interior inclusion, parameter $\alpha$ is 0.5 , and $\sigma$ takes the form of $\sigma_{\max }\left(1-\frac{d_{\mathrm{lnc}}}{r}\right)$, with $r$ being the radius of cross section, $d_{\mathrm{Inc}}$ the depth of the inclusion away from fracture surface, and $\sigma_{\max }$ the maximum stress at specimen surface. 

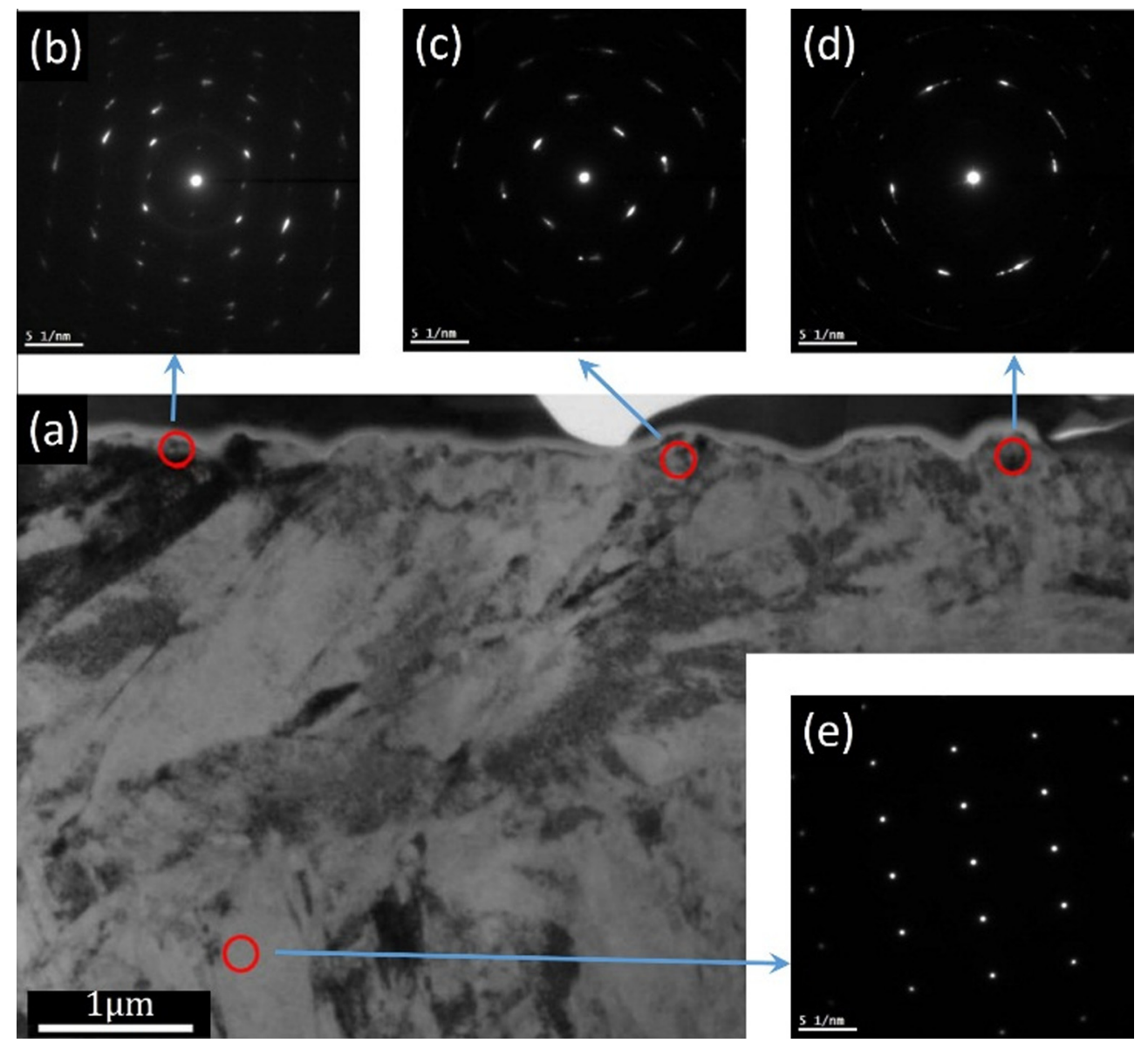

Fig. 8. TEM observations with SAD examinations of specimen A1. (a) TEM image showing microstructure of fracture surface profile, (b)-(d) SAD patterns just underneath fracture surface, and (e) SAD pattern away from fracture surface.
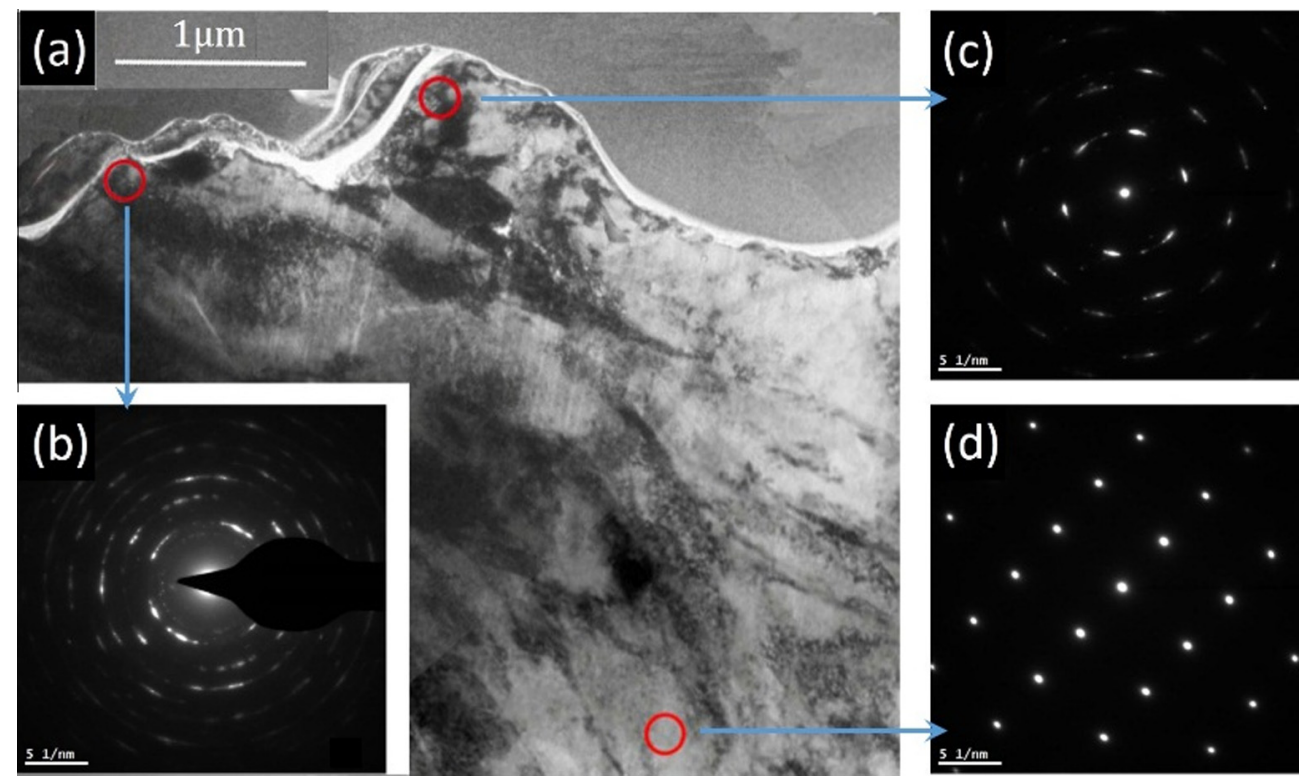

Fig. 9. TEM observations with SAD examinations of specimen A2. (a) TEM image showing microstructure of fracture surface profile, (b) and (c) SAD patterns just underneath fracture surface, and (d) SAD pattern away from fracture surface.

The values of stress intensity factor ranges for $\mathrm{FGA}\left(\Delta K_{\mathrm{FGA}}\right)$ and fish-eye $\left(\Delta K_{\mathrm{FiE}}\right)$ were calculated similarly. For a surface defect, area takes the value of surface defect projection area, $\alpha$ is 0.65 , and $\sigma$ equals to the maximum stress at specimen surface.
The calculated results of $\Delta K$ values are shown in Fig. 13. It is seen that the value of $\Delta K_{\text {Def }}$ is much larger than that of $\Delta K_{\text {Inc }}$ or $\Delta K_{\mathrm{FGA}}$ at a given applied maximum stress ( $\Delta K_{\text {Def }}$ is the $\Delta K$ value for induced surface defect). The large value of $\Delta K_{\text {Def }}$ is responsible 


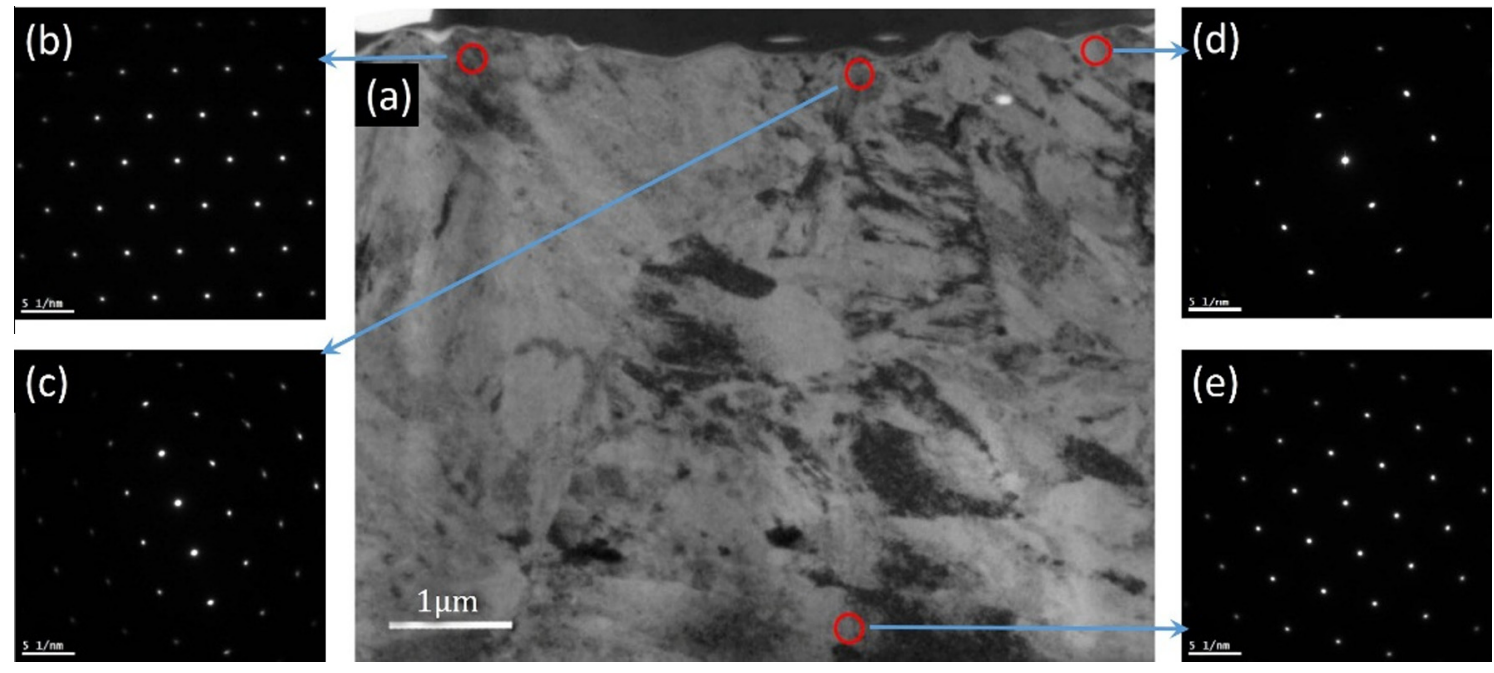

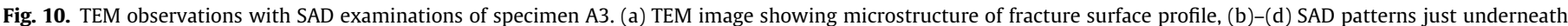
fracture surface, and (e) SAD pattern away from fracture surface.

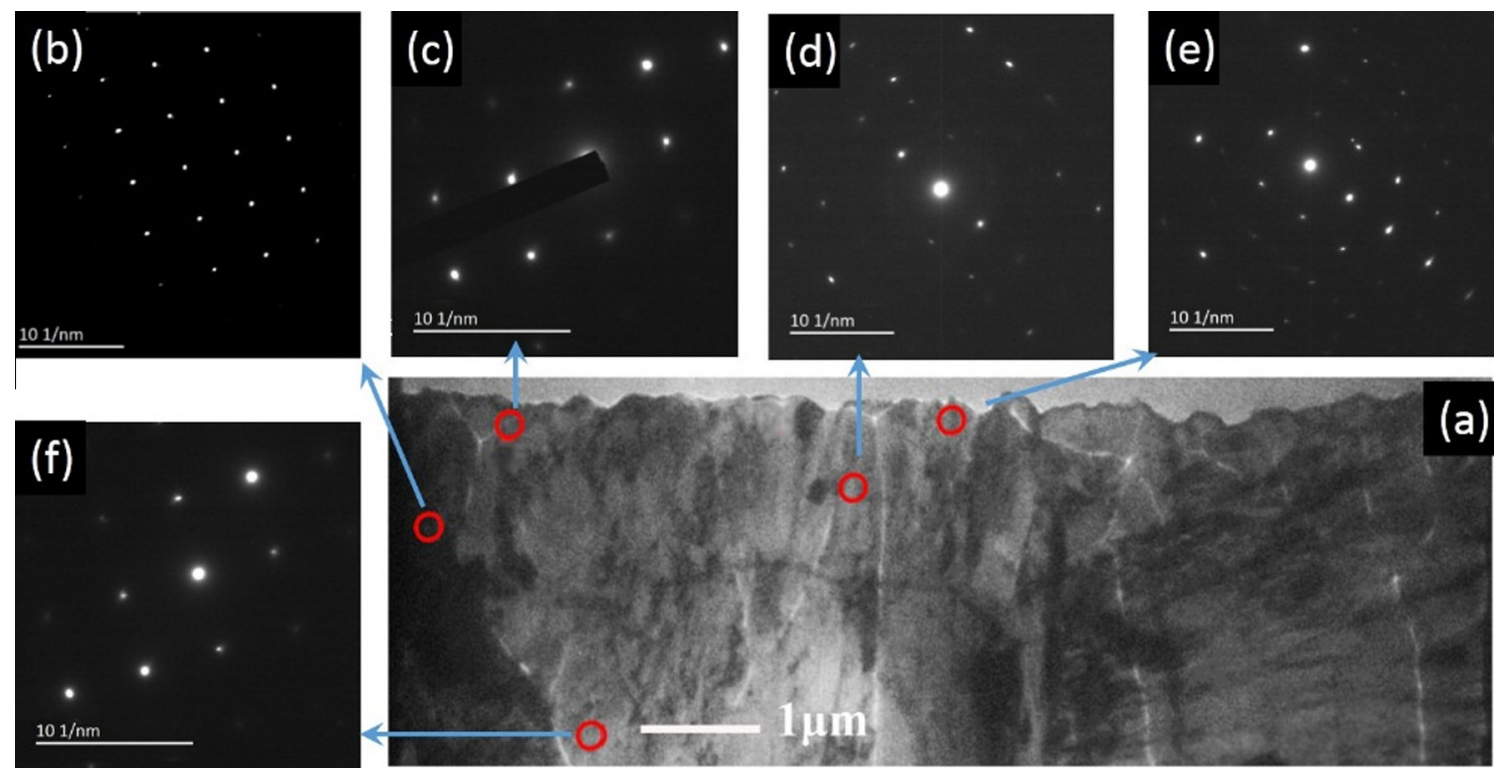

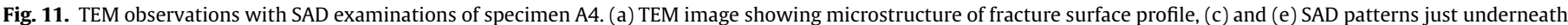
fracture surface, (d), (b) and (f) SAD patterns gradually away from fracture surface.

for the crack initiation from the induced surface defects of group B specimens.

Here, we adopted the models proposed by Murakami [33] and by Shyam et al. [35] to interpret the effect of induced surface defect on the degradation of fatigue strength. The model proposed by Murakami [33] is

$\sigma_{\mathrm{W}}=1.43(\mathrm{Hv}+120) /(\sqrt{\text { area }})^{1 / 6}$,

where $\sigma_{\mathrm{W}}$ is the fatigue limit (fatigue strength), Hv is the Vickers hardness, and area is the projected area on the plane perpendicular to the loading direction. Recently, Shyam et al. [35] also proposed a model based on elastic plastic fracture mechanics (EPFM model) for predicting fatigue limit $\left(\sigma_{\mathrm{W}}\right)$ in the presence of submillimeter holes, which is

$\sigma_{\mathrm{W}}=\frac{2 \sigma_{0}}{\pi} \arccos \left[\exp \left(\frac{-\pi E}{32 \sigma_{0} a}\right)\right]$ where $\sigma_{0}$ is the flow stress, which is the arithmetic mean of the yield strength and the ultimate tensile strength, $E$ is the Young's modulus, and $a$ is half of the surface crack length.

Based on Eqs. (3) and (4), we calculated the values of fatigue limit (fatigue strength at $10^{7}$ cycles) of the present case and several cases in literature [34,42-44], and the calculated values were normalized by experimental data. These results are shown in Fig. 14 with Fig. 14a based on Murakami model and Fig. 14b based on EPFM model.

It is seen that the present data together with the results from literature [34,42-44] are reasonably consistent within a scatter band described by both models. The scatter band of Fig. 14a is between 0.82 and 0.98 , and that of Fig. 14b is between 0.98 and 1.35. It is noted that the predictions by Murakami model are of a feature of underestimation for experimental data, whereas the predictions by EPFM model are of a feature of overestimation for experimental results. It seems that both models may reasonably 


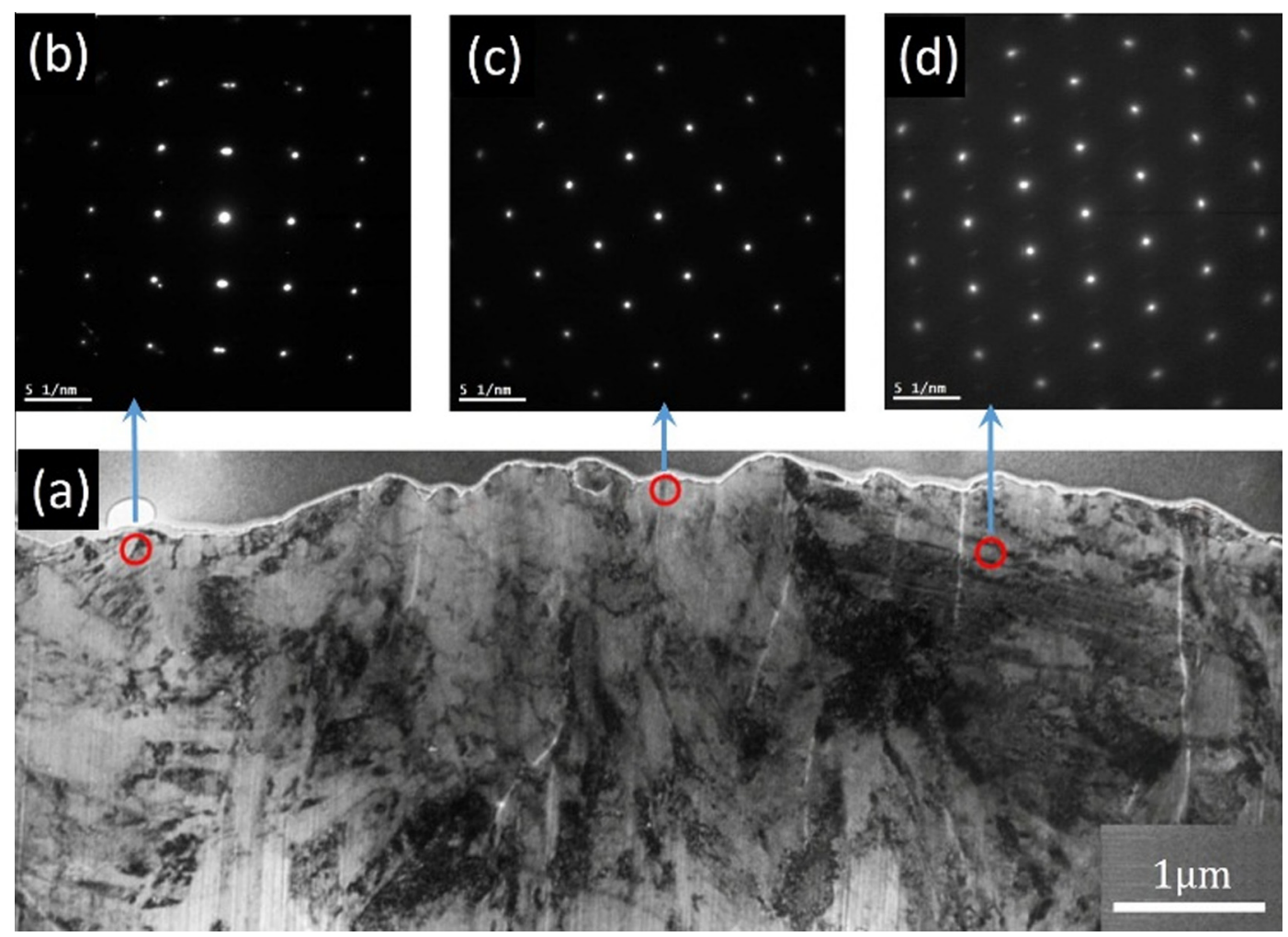

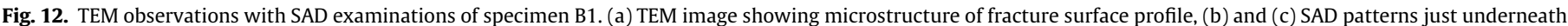
fracture surface, and (d) SAD pattern away from fracture surface.

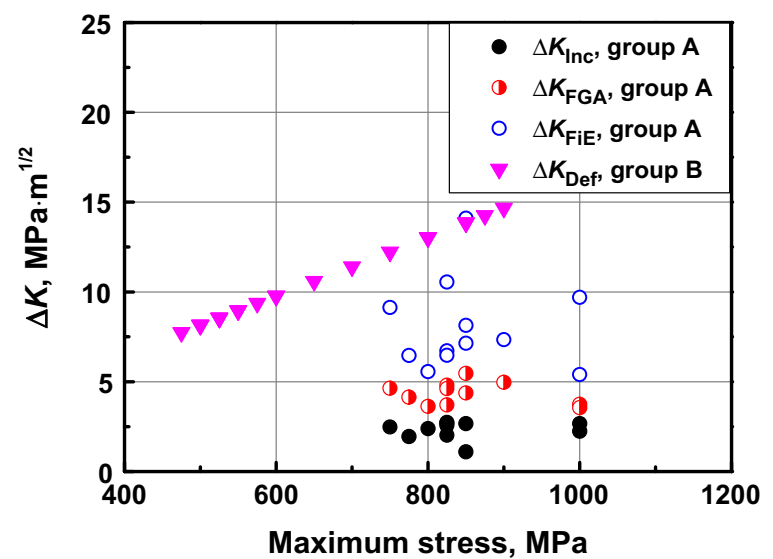

Fig. 13. Stress intensity factor range for inclusion, FGA and fish-eye of group A specimens and for induced surface defects of group B specimens as a function of applied maximum stress.

describe the effect of surface defect on the degradation of fatigue resistance and estimate the resulted fatigue strength. However, it should be noted that the crack or the defect size described by both models shown in Fig. 14a and b is in a range between $100 \mu \mathrm{m}$ and $300 \mu \mathrm{m}$. If the crack or defect size is outside this range, the predictions are likely to deviate far from unity, i.e. a large deviation may present between prediction values and related experimental data. It seems that for the case of defect size smaller than $100 \mu \mathrm{m}$, the defect is too small to become a dominant crack origin, and for the case of defect size lager than $300 \mu \mathrm{m}$ (e.g. hole diameter), the defect is too large to be regarded as a crack.

One may note that the fatigue testing of present investigation was via rotary bending at the frequency of $52.5 \mathrm{~Hz}$. For the loading cycles extended to VHCF regime, the method of ultrasonic vibration (normally with the frequency of $20 \mathrm{kHz}$ ) has been commonly used to substantially accelerate the testing process (e.g. [7,17,4547]). The use of ultrasonic vibration method will more or less cause the frequency effect on the related fatigue behavior. Such effect may vary with respect to the lattice type and the strength level of test materials $[7,17,45-47]$.

\subsection{Crack interior initiation from inclusion}

It has been mentioned that most specimens of group A failed in the mode of crack interior initiation from an inclusion beyond $5 \times 10^{6}$ cycles of cyclic loading (Fig. 2). Observations for such specimens showed the crack initiation region of FGA morphology. However, the FGA region is not well developed for the cases of fatigue life between $10^{7}$ and $10^{8}$ cycles compared with the cases of the fatigue life beyond $10^{8}$ cycles. As shown in Fig. 15, the morphology of FGA region is clear and with an evidently large area at the fatigue life of $1.6 \times 10^{8}$ (Fig. 15b) compared to that at the fatigue life of $2.1 \times 10^{7}$ (Fig. 15a).

The TEM observations further indicate that the well-developed FGA region at the fatigue life of $1.6 \times 10^{8}$ is a layer of nanograins (Fig. 9b and c). While for the FGA region which was less developed (Fig. 7a), the spots of SAD patterns were just slightly elongated (Fig. 8b-d), indicating that the grain refinement was insufficient. This result is in agreement with our recently proposed NCP model [31] that sufficient pressing process between originated crack surfaces is necessary for the formation of the nanograins. It is noted that, the TEM observations for the FGA at the fatigue life of $1.6 \times 10^{8}$ cycles (Fig. 9) also indicate that the nanocrystalline layer is thinner compared with the situation in high carbon steels [31]. This may be caused by the different volume fractions of retained austenite between a medium carbon steel and a high carbon steel. During the process of fatigue crack initiation and early growth, the 

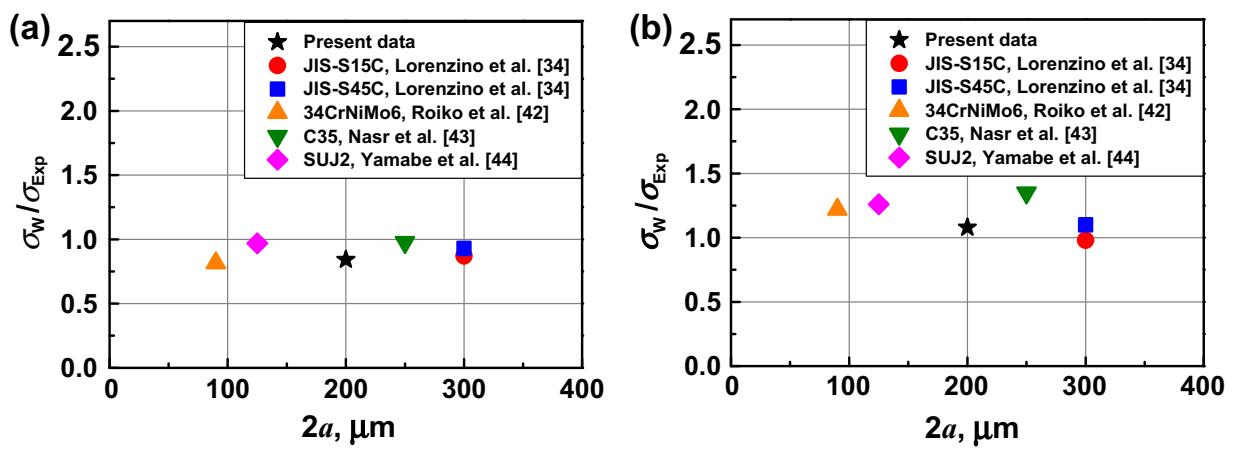

Fig. 14. Normalized fatigue strength at $10^{7}$ cycles $\left(\sigma_{\mathrm{w}} / \sigma_{\mathrm{Exp}}\right)$ as a function of defect size $2 a$, based on Murakami model (a), and on EPFM model (b).
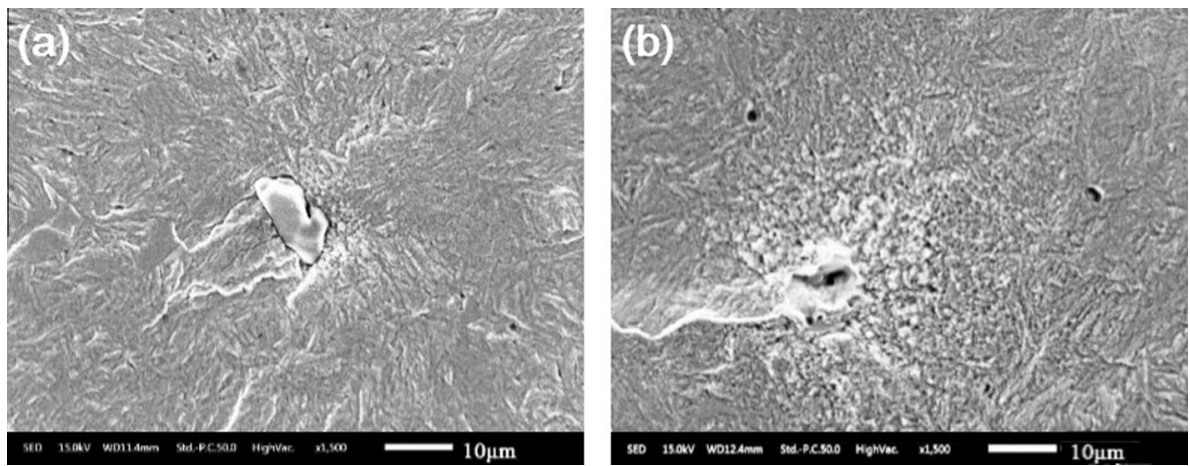

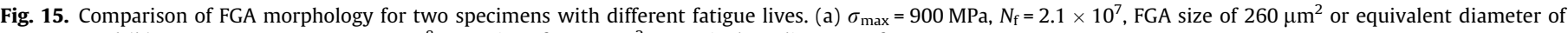
$18.2 \mu \mathrm{m}$, and (b) $\sigma_{\max }=850 \mathrm{MPa}, N_{\mathrm{f}}=1.6 \times 10^{8}$, FGA size of $1880 \mu \mathrm{m}^{2}$ or equivalent diameter of $48.9 \mu \mathrm{m}$.

energy produced by local plastic deformation and by crack surface formation may cause the transformation of retained austenite (fcc lattice) to tempered microstructure (bcc lattice) in the crack wake, so that to result in a volume expansion in the local region. Such a volume expansion is one of the important factors to increase the possibility of the contacting between two crack surfaces and therefore to cause crack closure in the NCP process; and the volume expansion is mainly provided by the transformation of retained austenite in steels. For the medium carbon steel used in this paper, the possible volume fraction of retained austenite was smaller than that in high carbon steels, so that the crack surface contacting (and therefore the grain refinement) was not as substantial as that in the situation of high carbon steels.

\subsection{Crack interior initiation from grain boundary}

The fracture surface observations on group A specimens showed that crack initiation from the interior of specimen in VHCF regime. Inclusions within the control volume [3] are the majority of the weakest spots for crack initiation, and in some rare cases, grain boundary as a kind of matrix inhomogeneities may also be the possible location for crack initiation. For group A, 2 out of 12 specimens that failed by crack interior initiation were due to grain boundary as crack origin. This implies that crack initiation mode for VHCF is not only the competition between surface type and interior type, but also the competition between inclusion initiation and grain boundary initiation for the interior type. Normally, the interface between a relatively large inclusion and matrix is more prone to debond, thus inclusions are the preferred origins for crack interior initiation. However, the control volume of specimen for rotary bending is relatively smaller than that for axial loading [3]. Therefore, the probability of inclusion initiation for rotary bending method will be restricted, and grain boundary especially the triple point like the case of Fig. 5 will increase the possibility for being the crack origin to cause fatigue failure in VHCF regime.

For the case of grain boundary as crack origin in VHCF regime, the morphology of crack initiation region is of rough surface like FGA (Fig. 5). The observations on the profiles of specimens A3 and A4 (crack initiation due to grain boundary) showed that the microscopic feature of the crack initiation region is without the evidence of grain refinement (Figs. 10 and 11), which differs from what happened in the case of inclusion as crack origin to form an FGA region (a layer of nanograins). The possible reason is that for the debonding of grain boundary like a triple point, a dis-match between the two parts of debonded grain boundary may be incurred due to the volume expansion (retained austenite transformation) and the slightly displacement (relaxation of residual stress) at the initiation location, which will retard the contacting of the originated crack surfaces and the process of grain refinement.

\subsection{Crack initiation from induced surface defects}

The fractography observations of the specimens with induced surface defects showed that all specimens failed due to crack initiation from the surface defects. This indicates that the surface defect size of $200 \mu \mathrm{m}$ in diameter and $200 \mu \mathrm{m}$ in depth is an overwhelming source responsible for crack initiation. As plotted in Fig. 13 , the $\Delta K$ values for surface defects are evidently larger than those for inclusions, FGAs and even fish-eyes, i.e. the $\Delta K$ value or the driving force for crack initiation pertained by a surface defect is large enough to be the predominant site for crack initiation from low cycle fatigue to VHCF regimes, and the related fatigue strength is substantially degraded. If expressed in terms of fatigue life, the value is reduced by more than two orders of magnitude at the applied maximum stress of $800 \mathrm{MPa}$ (Fig. 2). It is vital to note that 
if an engineering component contains a surface defect with a considerable size due to different reasons, the estimated fatigue life will be remarkably reduced.

The examinations on the profile of crack initiation region originated from surface defects showed that there is no evidence of grain refinement even experienced VHCF process (Fig. 12), which differs from the case of crack interior initiation from an inclusion, for which the initiation characteristic region of FGA is a nanograin layer. The fracture surface of crack initiation region due to surface defects remains coarse microstructure and is without the evidence of grain refinement, which may be explained as the follows. For the case of inclusion induced crack interior initiation, the sizes of the characteristic region for crack initiation of FGA are within a certain range, the related $\Delta K$ values are also within a certain range, and the crack growth rate is extremely slow [24]. For the present case of crack initiation from surface defects, the defect size is evidently larger than that of common FGA, the related $\Delta K$ values are considerably larger than those pertained by FGA (Fig. 13), and the related crack growth rate will be much faster than that of FGA crack. Thus the condition for FGA formation does not maintain and the initiation characteristics differ from the case of crack interior initiation due to inclusions.

It is obvious that for the specimens with induced surface defects (drill holes), the severe location of stress concentration is at the root of the drill hole, which is the location of fatigue crack initiation. For the specimens that failed at high-cycle fatigue and VHCF regimes, the cycling stress that causes failure is quite small. Evidence of plastic strain at the root of the notch is difficult to measure using conventional methods. The local strain at the defect root is an important issue and is a topic of future investigation.

\section{Conclusions}

The conclusions of this investigation for a structural steel with medium carbon content are drawn as follows:

(1) For the specimens with induced surface defects, the fatigue strength decreased by about $40 \%$ in high-cycle fatigue and VHCF regimes.

(2) The effect of induced surface defect on the degradation of fatigue strength can be predicted by Murakami model and EPFM model for the defect size between $100 \mu \mathrm{m}$ and $300 \mu \mathrm{m}$.

(3) For the case of crack initiation from an inclusion, the region of FGA is a layer of nanograins. The FGA region is well developed in the specimens with the fatigue life beyond $10^{8}$ cycles and is less developed in the specimens with the fatigue life below $10^{8}$ cycles. For the case of crack initiation from grain boundary, the FGA-like morphology in the crack initiation region is just a rough area but is not nanograin layer due to less contacting between the originated crack surfaces.

(4) For the specimens with induced surface defects, crack initiation is from the surface defect due to the large driving force at the root of surface defect. The microstructure underneath the fracture surface of crack initiation region remains coarse grains even in VHCF regime, which is a result of insufficient contact between the originated crack surfaces.

\section{Acknowledgements}

The authors would like to appreciate the financial supports from the National Basic Research Program of China
(2012CB937500) and from the National Natural Science Foundation of China (11572325).

\section{References}

[1] Naito T, Ueda H, Kikuchi M. Fatigue behavior of carburized steel with internal oxides and nonmartensitic microstructure near the surface. Metall Trans 1984;15A:1431-6.

[2] Marines-Garcia I, Paris P, Tada H, Bathias C. Fatigue crack growth from small to long cracks in VHCF surface initiations. Int J Fatigue 2007;29:2072-8.

[3] Lei Z, Xie J, Sun C, Hong Y. Effects of loading condition on very-high-cycle fatigue behavior and dominant variable analysis. Sci China-Phys Mech Astron 2014;57:74-82.

[4] Sun C, Liu X, Hong Y. A two-parameter model to predict fatigue life of highstrength steels in a very high cycle fatigue regime. Acta Mech Sin 2015;31:383-91.

[5] Zimmermann M. Diversity of damage evolution during cyclic loading at very high numbers of cycles. Int Mater Rev 2012;57:73-91.

[6] Li SX. Effects of inclusions on very high cycle fatigue properties of high strength steels. Int Mater Rev 2012;57:92-114.

[7] Mayer H. Recent developments in ultrasonic fatigue. Fatigue Fract Eng Mater Struct 2016;39:3-29.

[8] Hong Y, Zhao A, Qian G. Essential characteristics and influential factors for very-high-cycle fatigue behavior of metallic materials. Acta Metall Sin 2009;45:769-80.

[9] Wang Q, Bathias C, Kawagoishi N, Chen Q. Effect of inclusion on subsurface crack initiation and gigacycle fatigue strength. Int J Fatigue 2002;24:1269-74.

[10] Shiozawa K, Morii Y, Nishino S, Lu L. Subsurface crack initiation and propagation mechanism in high strength steel in a very high cycle fatigue regime. Int J Fatigue 2006;28:1521-32.

[11] Makino T. The effect of inclusion geometry according to forging ratio and metal flow direction on very high-cycle fatigue properties of steel bars. Int J Fatigue 2008;30(8):1409-18.

[12] Umezawa O, Nagai K. Deformation structure and subsurface fatigue crack generation in austenitic steels at low temperature. Metall Mater Trans 1998;29A:809-22.

[13] Chai G. The formation of subsurface non-defect fatigue crack origins. Int J Fatigue 2006;28:1533-9.

[14] Lei Z, Hong Y, Xie J, Sun C, Zhao A. Effects of inclusion size and location on veryhigh-cycle fatigue behavior for high strength steels. Mater Sci Eng A 2012;558:234-41.

[15] Zhou C, Qian G, Hong Y. Fractography and crack initiation of very-high-cycle fatigue for a high carbon low alloy steel. Key Eng Mater 2006;324325:1113-6.

[16] Qian G, Hong Y, Zhou C. Investigation of high cycle and very-high-cycle fatigue behaviors for a structural steel with smooth and notched specimens. Eng Fail Anal 2010;17:1517-25.

[17] Sakai T, Sato Y, Oguma N. Characteristic S-N properties of high-carbonchromium-bearing steel under axial loading in long-life fatigue. Fatigue Fract Eng Mater Struct 2002;25:765-73.

[18] Qian G, Zhou C, Hong Y. Experimental and theoretical investigation of environmental media on very-high-cycle fatigue behavior for a structural steel. Acta Mater 2011;59:1321-7.

[19] Qian G, Hong Y. Effects of environmental media on high cycle and very-highcycle fatigue behaviors of structural steel $40 \mathrm{Cr}$. Acta Metall Sin 2009;45:1356-63.

[20] Hong Y, Zhao A, Qian G, Zhou C. Fatigue strength and crack initiation mechanism of very-high-cycle fatigue for low alloy steels. Metall Mater Trans A 2012;43:2753-62.

[21] Qian G, Zhou C, Hong Y. A model to predict S-N curves for surface and subsurface crack initiations in different environmental media. Int J Fatigue 2015;71:35-44.

[22] Bathias C, Paris PC. Gigacycle fatigue in mechanical practice. New York: Marcel Dekker; 2005.

[23] Yang Z, Li S, Liu Y, Li G, Hui W, Weng Y. Estimation of the size of GBF area on fracture surface for high strength steels in very high cycle fatigue regime. Int J Fatigue 2008;30:1016-23.

[24] Hong Y, Lei Z, Sun C, Zhao A. Propensities of crack interior initiation and early growth for very-high-cycle fatigue of high strength steels. Int J Fatigue 2014;58:144-51.

[25] Murakami Yu, Nomoto T, Ueda T, Murakami Ya. On the mechanism of fatigue failure in the superlong life regime ( $N>10^{7}$ cycles), Part II: a fractographic investigation. Fatigue Fract Eng Mater Struct 2000;23:903-10.

[26] Murakami Yu, Nomoto T, Ueda T, Murakami Ya. On the mechanism of fatigue failure in the superlong life regime $\left(N>10^{7}\right.$ cycles), Part I: influence of hydrogen trapped by inclusions. Fatigue Fract Eng Mater Struct 2000;23:893-902.

[27] Sakai T. Review and prospects for current studies on very high cycle fatigue of metallic materials for machine structural use. J Solid Mech Mater Eng 2009;3:425-39.

[28] Sakai T, Oguma N, Morikawa A. Microscopic and nanoscopic observations of metallurgical structures around inclusions at interior crack initiation site for a bearing steel in very high-cycle fatigue. Fatigue Fract Eng Mater Struct 2015;38:1305-14. 
[29] Grad P, Reuscher B, Brodyanski A, Kopnarski M, Kerscher E. Mechanism of fatigue crack initiation and propagation in the very high cycle fatigue regime of high-strength steels. Scr Mater 2012;67:838-41.

[30] Shanyavskiy AA. Mechanisms and modeling of subsurface fatigue cracking in metals. Eng Fract Mech 2013;110:350-63.

[31] Hong Y, Liu X, Lei Z, Sun C. The formation mechanism of characteristic region at crack initiation for very-high-cycle fatigue of high-strength steels. Int J Fatigue 2016;89:108-18.

[32] Li W, Sakai T, Wakita M, Mimura S. Influence of microstructure and surface defect on very high cycle fatigue properties of clean spring steel. Int J Fatigue 2014;60:48-56.

[33] Murakami Yukitaka. Metal fatigue: effects of small defects and nonmetallic inclusions. London: Elsevier; 2002.

[34] Lorenzino P, Okazaki S, Matsunaga H, Murakami Y. Effect of small defect orientation on fatigue limit of carbon steels. Fatigue Fract Eng Mater Struct 2015:38:1076-86.

[35] Shyam A, Blau P, Jordan T, Yang N. Effect of submillimeter size holes on the fatigue limit of a high strength tool steel. Fatigue Fract Eng Mater Struct 2014:37:368-79.

[36] Endo M, Ishimoto I. The fatigue strength of steels containing small holes under out-of-phase combined loading. Int J Fatigue 2006;28:592-7.

[37] Fernández-Pariente I, Bagherifard S, Guagliano M, Ghelichi R. Fatigue behavio of nitrided and shot peened steel with artificial small surface defects. Eng Fract Mech 2013;103:2-9.
[38] Morel F, Guerchais R, Saintier N. Competition between microstructure and defect in multiaxial high cycle fatigue. Frattura ed Integrita 2015;33:404-14.

[39] Leopold G, Nadot Y, Billaudeau T, Mendez J. Influence of artificial and casting defects on fatigue strength of moulded components in Ti-6Al-4V alloy. Fatigue Fract Eng Mater Struct 2015;38:1026-41.

[40] Williams DB, Carter CB. Transmission electron microscopy: a textbook for materials science. 2nd ed. New York: Springer; 2009.

[41] Murakami Y, Kodama S, Konuma S. Quantitative evaluation of effects of nonmetallic inclusions on fatigue strength of high strength steel. Trans Jpn Soc Mech Eng A 1988;54:688-96.

[42] Roiko A, Solin J, Murakami Y. Inclined defects and their effect on the fatigue limit and small crack growth. In: MATEC web of conferences, vol. 12; 2014. p. 07002.

[43] Nasr A, Nadot Y, Bouraoui C, Fathallah R, Jouiad M. Fatigue initiation in C35 steel: influence of loading and defect. Int J Fatigue 2010;32:780-7.

[44] Yamabe J, Matsui T. Influence of hardness, defect size and retained austenite on fatigue strength at $N=10^{7}$ for carburized steels. J Soc Mater Sci Jpn 2007;56 (3):236-43.

45] Berger C, Pyttel B, Schwerdt D. Beyond HCF - is there a fatigue limit? Materialwiss Werkstofftech 2008;39:769-76.

[46] Bathias C. Gigacycle fatigue properties of bearing steels. J ASTM Int 2010;7: JAI102712.

[47] Zhao A, Xie J, Sun C, Lei Z, Hong Y. Effects of strength level and loading frequency on very-high-cycle fatigue behavior for a bearing steel. Int J Fatigue 2012;38:46-56. 